\title{
Linking major depression and the neural substrates of associative processing
}

\author{
Eiran Vadim Harel ${ }^{1} \cdot$ Robert Langley Tennyson ${ }^{2,3} \cdot$ Maurizio Fava $^{4} \cdot$ Moshe Bar $^{5}$
}

Published online: 23 August 2016

(C) Psychonomic Society, Inc. 2016

\begin{abstract}
It has been proposed that mood correlates with the breadth of associative thinking. Here we set this hypothesis to the test in healthy and depressed individuals. Generating contextual associations engages a network of cortical regions including the parahippocampal cortex (PHC), retrosplenial complex, and medial prefrontal cortex. The link between mood, associative processing, and its underlying cortical infrastructure provides a promising avenue for elucidating the mechanisms underlying the cognitive impairments in major depressive disorder (MDD). The participants included 15 nonmedicated individuals with acute major depressive episodes and 15 healthy matched controls. In an fMRI experiment, participants viewed images of objects that were either strongly or weakly associated with a specific context (e.g., a beach chair vs. a water bottle) while rating the commonality of each object. Analyses were performed to examine the brain activation and structural differences between the groups.
\end{abstract}

Electronic supplementary material The online version of this article (doi:10.3758/s13415-016-0449-9) contains supplementary material, which is available to authorized users.

Eiran Vadim Harel

eiranharel@gmail.com

1 Beer Yaakov Mental Health Center affiliated with the Sackler Faculty of Medicine, Tel Aviv University, Tel Aviv, Israel

2 Department of Anthropology, University of Washington, Seattle, WA, USA

3 Center for Studies of Demography and Ecology, University of Washington, Seattle, WA, USA

4 Division of Clinical Research, Massachusetts General Hospital Research Institute, Boston, MA, USA

5 Gonda Center for Brain Research, Bar-Ilan University, Ramat Gan, Israel
Consistent with our hypothesis, controls showed greater activation of the contextual associations network than did depressed participants. In addition, PHC structural volume was correlated with ruminative tendencies, and the volumes of the hippocampal subfields were significantly smaller in depressed participants. Surprisingly, depressed participants showed increased activity in the entorhinal cortex (ERC), as compared with controls. We integrated these findings within a mechanistic account linking mood and associative thinking and suggest directions for the future.

Keywords Depression · Context · Parahippocampus · Associations $\cdot$ Entorhinal $\cdot$ Rumination

The notion that our thinking is associative in nature has long been discussed and studied (Barsalou, 1999; Moores, Laiti, \& Chelazzi, 2003). Bridging separate concepts through associations is the basis of cognition, via processes such as learning, memory, and the progression of thought. In a series of studies, Bar and colleagues have found evidence for a network of cortical regions that mediate contextual associative processing (Bar, 2007, 2009a, b; Bar \& Aminoff, 2003). This network is active when participants are presented with visual images of objects that are strongly associated with a specific context (e.g., a bowling pin) relative to objects that are equally common in our environment but are not strongly associated with any specific context (e.g., a fly). The brain regions involved in this contextual processing network include the retrosplenial complex (RSC), the parahippocampal cortex (PHC), and the medial prefrontal cortex (MPFC) (Aminoff, Schacter, \& Bar, 2008; Bar, 2004; Bar \& Aminoff, 2003). It has been proposed that the RSC activates the gist of a context frame, whereas the PHC activates the specific perceptual features and associated items contained within it (Aminoff et al., 2008). The MPFC's 
suggested function is to generate expectations and predictions about what is going to occur in the immediate environment, based on information activated within the context frame (Bar, 2004; Bar, Aminoff, Mason, \& Fenske, 2007; Herbst, Kveraga, \& Bar, Manuscript submitted for publication).

It has been hypothesized (Bar \& Ullman, 1996; Mandler \& Johnson, 1976; Minsky, 1975; Schank, 1975) that context frames facilitate the retrieval of information, whereby topdown information (e.g., context) or bottom-up information (e.g., low spatial frequencies; Bar, 2004; Oliva \& Torralba, 2001) about the perceived stimulus can activate associated information within the context frame, increasing the expectancy for specific stimuli. Thus, the availability of internally generated, associated information accompanying a stimulus impacts subsequent processing of information.

Mood is another construct that has been documented to influence the encoding and retrieval of associated items. Notably, studies indicate that positive mood broadens the scope of associations and loosens the conceptual relations between associations (Isen \& Daubman, 1984; Isen, Johnson, Mertz, \& Robinson, 1985). Positive affective states do not only evoke the generation of more unique, broadly related associates in free-association tasks, but they also lead to more remote categorical associations (e.g., surfboard = a vehicle) (Isen \& Daubman, 1984; Rosch \& Mervis, 1975) and promote more creative problem solving, as measured, for example, by facilitated performance on the candle task (Duncker, 1945) and Remote Associates Test (Isen, Daubman, \& Nowicki, 1987; Mednick, Mednick, \& Jung, 1964; Mednick, Mednick, \& Mednick, 1964). Recently, it has been proposed (Bar, 2009a) and later demonstrated that the reverse is also true: increasing the breadth of associations can positively influence mood, indicating that the moodassociative processing relationship may be bidirectional (Mason \& Bar, 2012), which could potentially be used as method for alleviating mood. In addition, it was found that mental load directly influences the breadth of associations provided in a free-association task (Baror \& Bar, 2016), and as such it was suggested that ruminative thinking can be seen as mental load that accordingly limits associative scope.

Mood disorder patients display characteristic relationships between mood and thought that would be expected from these findings. For instance, patients in hypo-manic or manic states, characterized by elevated mood, often exhibit a loosening of associative links between concepts, which can lead to "flight of ideas" (Andreasen, 1979; Lake, 2008; Sass \& Pienkos, 2015) and increases in creativity (Davis, 2009). In more severe manic states, in which elevation of mood is accentuated, the extreme loosening of associations may lead to incoherence of thought (Andreasen 1979; Lake, 2008; Sass \& Pienkos, 2015). Conversely, depressed mood and anhedonia - the major features of major depressive disorder (MDD) — are characterized by ruminative thought pattern that is repetitive in nature and revolves around constrained and narrow themes (Andreasen, 1979; Lake, 2008; Nolen-Hoeksema, 2000; Sass \& Pienkos, 2015).

Much of the previous experimental research investigating mood and associative thought has employed mood induction techniques in healthy participants (Ferrer, Grenen, \& Taber, 2015), rather than focusing on psychiatric disorders, making the applicability of these findings to clinical populations unclear. Psychiatric disorders are characterized by more longterm and severe levels of emotional distress than could be simulated in a mood induction study. Investigating clinical populations that are characterized by specific affective states allows us to elucidate the neural networks related to changes in associative thinking and, furthermore, whether these networks are related to functional and morphological changes in the identified contextual associative network. By focusing on differences within this network, research could create specific targets for future treatments. For example, Mason and Bar (2012), found that experimentally increasing the breadth of associations may lead to improved mood in healthy individuals. If differences in the contextual associative network are associated with the thought patterns characteristic of mood disorders, regulating this pathway in clinical disorders may be a fruitful therapeutic goal.

Given the proposed link between mood and associativity (Bar, 2009a), we hypothesized that contextual associative processing would be compromised in MDD patients. To test this hypothesis, we used functional magnetic resonance imaging (fMRI) to measure brain activity during contextual associative processing of objects presented visually, and compared brain activation between patients diagnosed with MDD and healthy participants. We predicted that MDD patients would display reduced activity in the contextual associative network when processing contextual information, compared with healthy controls. Building on previous studies demonstrating that morphological differences between MDD patients and healthy controls are often related to behavioral differences (e.g., Guo, Gatchel, \& Sahay, 2015), we also predicted that regions connected to contextual associative processing would show differences in gray matter volume. Furthermore, because of its explicit connection to both MDD and associative thinking, we also predicted that ruminative tendency would be associated with differences of functional activation within the contextual associations network, as well as with differences in gray matter volume. Finally, we predicted that our depressed participants would display selective decreases in hippocampal subfield volumes in accordance with previous studies showing reduced hippocampal subfields volumes in MDD, specifically the dentate gyrus (DG), and data showing direct links between associations and the hippocampal complex (Mayes, Montaldi, \& Migo, 2007; Travis et al., 2015; Treadway et al., 2015).

The DG subfield of the hippocampus is specifically responsible for transforming similar memories and patterns into 
distinct mental representations (pattern separation; Guo et al., 2015), which may play a role in connecting associated contextual representations. Therefore, we predicted that we would find decreased DG volume in MDD patients if they displayed our predicted activation differences in the contextual associative network. Furthermore, the DG has been linked with adult neurogenesis (Cameron \& Gould, 1994), which has been suggested to be reduced in mood disorders (Treadway et al., 2015) and to be upregulated with remission of symptoms mediated by SSRIs or psychotherapy (Guo et al., 2015). Although the link between associations, DG and depressionrelated reduction in neurogenesis is speculative, showing such a link empirically here will provide critical support for this hypothesis.

\section{Method and materials}

\section{Participants}

Fifteen adults diagnosed with MDD and fifteen healthy control participants were included in this study. Depressed participants were recruited through the Depression Clinical and Research Program (DCRP) of Massachusetts General Hospital and through an advertisement in the volunteer section of Craigslist. All participants went through a phone screening procedure and whoever appeared to meet the inclusion and exclusion criteria of the phone screening was scheduled for a full psychiatric SCID interview by a trained psychiatrist $(\mathrm{EVH})$. All participants provided informed consent in writing. The protocol was approved by the research ethics committee of Massachusetts General Hospital. All the included depressed participants met criteria for a DSM-IV diagnosis of a current major depressive episode, based on the Structured Clinical Interview for DSM-IV Disorders. Depressed individuals with psychotic features or participants taking psychotropic medications at the time or four weeks prior to the study or who met criteria for a current, comorbid diagnosis of any Axis I disorder, with the exception of social anxiety disorder, were not included in the study. Participants were evaluated using a clinician rated Hamilton rating scale of depression (HRSD), a 17-item scale for evaluating the severity of depressive symptoms, as well as the Quick Inventory of Depressive Symptoms- Self-Rated Questionnaire (QIDS-SR) and the Ruminative Response Style (RRS) questionnaire (Treynor, Gonzalez, \& Nolen-Hoeksema, 2003). The RRS is a 22-item, self-report measure of self-focused rumination about depressive mood, its causes, and consequences.

A group of healthy control participants, comparable to the experimental group in age, sex, and education, was recruited for this study using an advertisement in the volunteer section of Craigslist. Participants were excluded if they had any lifetime Axis I disorder or were suffering from any medical condition, acute or chronic. The demographic data collected are depicted in Table 1. After a complete description of the study to the participants, written informed consent was obtained.

For the functional analysis, we removed runs in which movement exceeded $2 \mathrm{~mm}$ in any direction. In all, four runs from three participants were excluded in the depressed group, and two runs from two participants were excluded in the healthy control group.

\section{Task design}

For the paradigm (see Fig. 1) we employed a rapid, eventrelated design. The target images were color photographs $(256 \times 256$ pixels $)$ of objects (69 strongly contextually associated, 69 weakly contextually associated) presented in isolation on a white background. Each target image was presented briefly $(150 \mathrm{~ms})$ and immediately followed by a colorful mask presented for $100 \mathrm{~ms}$. A red fixation cross then appeared, signaling the start of the response period, and turned black after 1,500 ms, signaling the end of the response period. The black fixation cross remained on the screen for the duration of the intertrial interval (ITI), which ranged from 200 to $9,250 \mathrm{~ms}$ (to allow a jittered ITI in multiples of the TR length [2,200 ms] and jittered stimulus presentation from the start of each TR). The fMRI session consisted of 138 unique trials, pseudorandomly ordered across the three functional runs, in addition to 28 practice trials that were completed prior to the main task (in the practice trials, we presented images not included in the main task). Each target image was only presented once within each session.

Table 1 Sample demographics

\begin{tabular}{llll}
\hline & Depressed Participants & Healthy Controls \\
\hline Age [years $(S D)]$ & $35.73(9.87)$ & $37(14.13)$ & $p=.78$ \\
Sex $(\mathrm{M} / \mathrm{F})$ & $7 / 8$ & $8 / 7$ & $/$ \\
Education $[$ years $(S D)]$ & $14.9(2.2)$ & $16.26(2.78)$ & $p=.14$ \\
QIDS score $(S D)$ & $22.1(6.6)$ & $2.7(2.6)$ & $29.1(6)$ \\
RSQ score $(S D)$ & $58.9(13)$ & $1.5(1.2)$ & \\
HRSD $(S D)$ & $21.8(4.7)$ & & \\
\hline
\end{tabular}




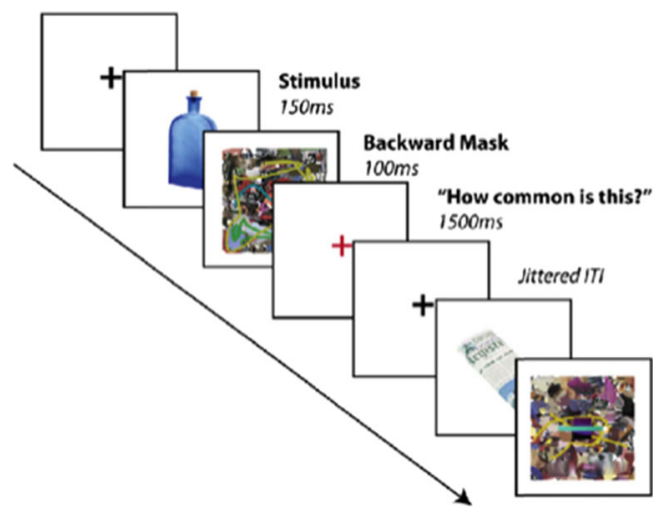

Fig. 1 Task timeline. Participants viewed objects presented briefly and in isolation $(150 \mathrm{~ms})$, followed by a colorful backward mask (100 ms), and then rated how common the object was (1.5-s response period). Between trials, participants viewed a black fixation cross for variable durations

Participants were instructed to rate how common each object was, on a three-point scale ranging from not common to common (e.g., a car would be rated common, whereas boxing gloves would be rated less common). This task was chosen because it requires high-level object recognition without focusing attention explicitly on the perceptual features or associative qualities of the object, an assumption that we later verified by comparing the commonality ratings across image conditions. Responses were provided on a three-button MRcompatible response box. The order of the three-point scale was counterbalanced across participants, to prevent confounds between rating and motor mapping, and participants practiced the appropriate mapping to proficiency before the practice trials began. Stimulus presentation and response collection was performed using the Psychophysics Toolbox (www. psychtoolbox.org) running on MATLAB software (www. mathworks.com), controlled by a MacBook Pro laptop with a monitor resolution of $1,024 \times 768$ and a refresh rate of 60 $\mathrm{Hz}$.

The images of strong and weak associative objects were compiled from a set previously normed and used in studies of contextual processing (Bar \& Aminoff, 2003; available at https://faculty.biu.ac.il/ barlab/context_localizer.html).

\section{Image acquisition}

Images were acquired using a Siemens 3-T Trio Tim MR magnet and a 32-channel RF head coil. We acquired functional image volumes as $\mathrm{T} 2 *$-weighted echoplanar images (EPIs) with the following parameters: 36 interleaved slices, 2,200-ms TR, 28-ms TE, 2.5-mm thickness, .75-mm gap, $64 \times 64$ matrix, 200-mm FOV (resulting in an in-plane voxel size of $3.125 \times 3.125 \times 2.5 \mathrm{~mm}$ ). Our fMRI sequence and slice prescription was optimized for reducing signal loss and distortion in the orbitofrontal cortex (on the basis of the recommendations of Deichmann, Gottfried, Hutton, \& Turner, 2003; Deichmann, Josephs, Hutton, Corfield, \&
Turner, 2002; Weiskopf, Hutton, Josephs, \& Deichmann, 2006 ), including the use of a modified $z$-shim prepulse moment and $30^{\circ}$ tilt of our slice prescription counterclockwise of the AC/PC line along the sagittal plane (Deichmann et al., 2003; Deichmann et al., 2002; Weiskopf et al., 2006). As a consequence of the limited slice prescription used in order to achieve optimal MPFC signal, the most dorsal portions of posterior parietal cortex were not captured in the scan volume for a majority of the participants. Each participant performed three functional runs, each consisting of 97 TRs. Each run included $11 \mathrm{~s}$ of fixation at the beginning (to allow for the fMRI signal to reach a steady state), and the corresponding five EPI volumes were discarded from further analysis. Each session included the acquisition of two high-resolution T1weighted multi-echo MPRAGE anatomical images (1-mm isotropic voxels), which were later averaged together.

\section{fMRI analysis}

Structural and functional imaging analyses were performed using the Freesurfer and FS-FAST analysis tools and processing stream developed at the Martinos Center for Biomedical Imaging (http://surfer.nmr.mgh.harvard.edu) and R statistical analysis software version 4.12.1 (R Development Core Team, 2011). Cortical reconstruction and volumetric segmentation of the T1-weighted scans were performed with Freesurfer 5.3 (http://surfer.nmr.mgh.harvard.edu/fswiki). The functionalimaging data wee preprocessed using the Freesurfer Functional Analysis Stream (FS-FAST), version 5.1 (http://surfer.nmr.mgh.harvard.edu/fswiki/FsFast). The images were motion-corrected (AFNI 3dvolreg; http://afni.nimh.nih. gov), slice-timing-corrected to the middle of the TR of a volume, intensity-normalized, and registered to the same participant's anatomical volume. Each 4-D functional dataset was resampled to a "common space" using the surface-based interparticipant registration created during the reconstruction of the cortical mantle that occurred as a portion of the Freesurfer processing pipeline (this brings the left and right cortical hemispheres into the average space of Freesurfer; fsaverage). Finally, 5-mm full-width-at-hald-maximum smoothing was applied to each surface (2-D surface-based smoothing).

A first-level general linear model (GLM) was set up consisting of two conditions/regressors of strong and weak context object processing. In addition to the task regressors and their temporal derivatives, estimated motion correction parameters and a set of polynomials (up to second degree) were included in the GLM as nuisance regressors. The first five volumes were removed from each fMRI run to allow for signal stabilization. The estimated hemodynamic response was defined by a gamma function of 2.25 -s hemodynamic delay and 1.25 -s dispersion. The data were then tested for statistical significance for each individual (first level), and contrast maps were constructed comparing the BOLD 
estimates for each condition. For the second-level analysis, both within-group (strong vs. weak context objects processing) and between-group (depressed vs. healthy control participants) whole-brain analyses were conducted.

A within-group analysis in the strong versus weak object contrast was done for the healthy control group and the depressed group using a voxel-wise threshold of $p<.001$, extent-thresholded to achieve a whole-brain, cluster-wise correction for multiple comparisons at $p<.01$. This was done to replicate previous findings of activation of the context network. Furthermore, a between-group comparison (depressed vs. controls) of the strong versus weak contrast was performed using a voxel-wise threshold of $p<.05$ and cluster-wise threshold of $p<.05$. Correction for multiple comparisons was done for the left hemisphere cortical surface. Finally, analyses testing the associations between the percent signal change functional activation and rumination scores as measured by the RRS questionnaire were also performed.

\section{Structural analysis}

To investigate the relationships between brain structure and MDD status, we conducted analyses of covariance (ANCOVAs) with the volume of the region of interest (ROI) as the outcome variable and MDD status as the grouping variable. We included total brain volume, age, sex, and years of education as covariates. Furthermore, to investigate relationships between brain structure and ruminative thinking, we performed linear regressions with the volume of the ROI as the outcome variable, the participant's score on the RRS survey as the explanatory variable, and total brain volume and MDD status as a covariate. ROIs included coordinates displaying activational differences in the functional analyses, regions that have been previously connected to the context network, and the hippocampal subfields. Significance levels are adjusted for multiple comparisons, where applicable - specifically, $p<$ .001 for hippocampal subfields and $p<.01$ for the nonhippocampal subfield group comparisons. One depressed participant was excluded from the structural analyses because of issues with autosegmentation and volume calculation.

\section{Results}

\section{fMRI results}

In the healthy control group $(n=15)$, the contrast between strong- and weak-context objects (contextual processing), using whole-brain analysis, showed cluster activations with the voxelwise threshold set to $p<.001$ (Fig. 2) and the cluster-wise threshold set to $p<.01$ in the RSC [comprising the precuneus $(-7.5,-53.4,2.5)$, fusiform $(-34.2-42.1-1.9)$, isthmus cingulate $(-4.7,-33.2,31.4)]$, PHC $(-3.7,-4.2,-5.8)$, and MPFC (-

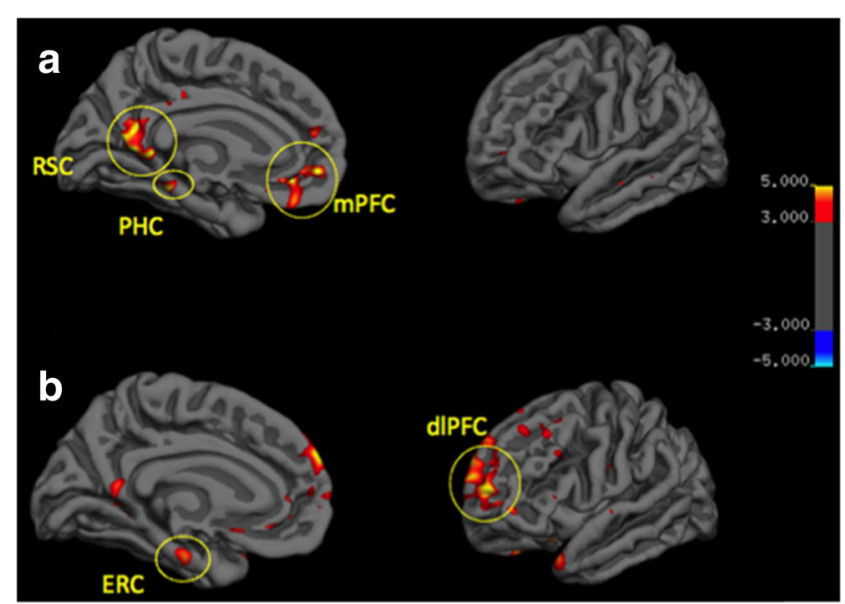

Fig. 2 Regions significantly associated with contextual processing in (a) healthy controls and (b) depressed patients (threshold $p<.001$ ). RSC, retrosplenial cortex; $\mathrm{PHC}$, parahippocampal cortex; $\mathrm{mPFC}$, medial prefrontal cortex; ERC, entorhinal cortex; dlPFC, dorsolateral prefrontal cortex

7.1, 5.7,-8.1); Fig. 2a]. These results replicate previous findings in healthy participants during contextual processing (Bar \& Aminoff, 2003; Bar, Aminoff, \& Schacter, 2008).

In the group of depressed patients, the contrast of contextual processing using whole-brain analysis showed cluster activations, with the voxel-wise threshold set to $p<.001$ and the cluster-wise threshold to $p<.01$, for the medial frontal gyrus (MFG) of the dorsolateral prefrontal cortex (DLPFC) $(-2.9$, $57.1,5.4)$ and the ERC $(-2.5,-18.1,-2.6)$ (Fig. 2b).

A group comparison using a voxel-wise threshold of $p<$ .05 and a cluster-wise threshold of $p<.05$, corrected for multiple comparisons for the left hemisphere cortical surface (depressed vs. healthy control participants) in the contrast of strong- versus weak-context objects, revealed differential activity in three regions after the correction: the MFG, PHC, and ERC (Fig. 3). A cluster in the left PHC (-18.3, -34.2, -9.6)

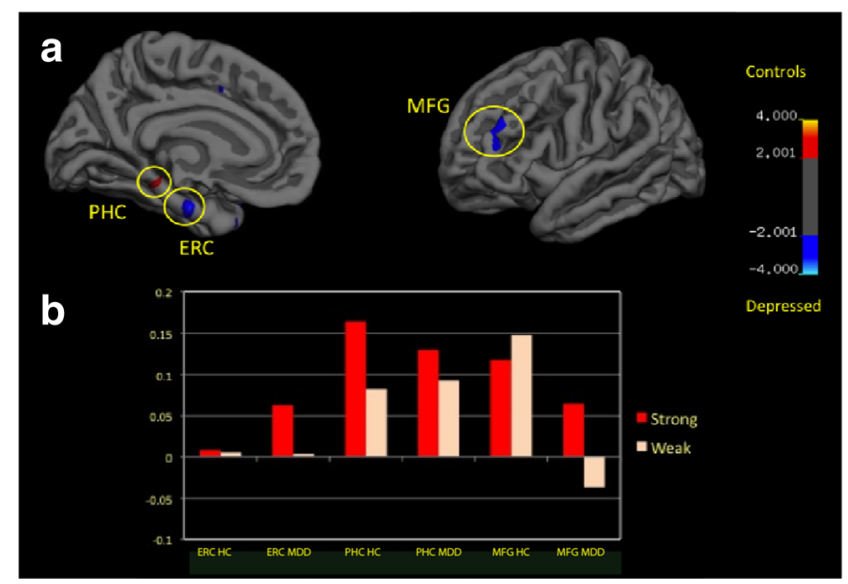

Fig. 3 As compared to healthy controls (HC), depressed patients (MDD) display reduced parahippocampal cortex (PHC), but increased entorhinal cortex (ERC) and medial frontal gyrus (MFG) differential activity during contextual processing $(p<.01)$. (a) Distribution of this differential activity. (b) Levels of activation (percent signal change) in these regions during the strong (color) and weak (white) contexts 
showed greater activation $(p<.05)$ in the healthy control than in the depressed group, and clusters in the ERC $(-19.8,-14.5$, $-23.6)$ and MFG $(-33.6,36.0,23.4)$ showed greater activity $(p$ $<.05)$ in the depressed than in the healthy control group (Fig. 3a). Percent signal change metrics from these functional clusters of differential activity were extracted for each participant in each condition (Fig. 3b).

Analyses testing the association between rumination and the contrast of contextual processing failed to find any significant associations. Finally, the behavioral component of our task, the reaction times of the commonality ratings and the ratings themselves, did not significantly differ between the healthy controls and the depressed patients.

\section{Structural results}

A group comparison (total, $n=29$; MDD participants, $n=14$ ) did not provide evidence of differences in the volumes of the coordinates showing activation differences (ERC, $F=0.02, p$ $>.10$; PHC, $F=0.13, p>.10$; MFG, $F=0.30, p>.10$ ). However, we did find a significant association between rumination scores and the gray matter volume of the coordinates located in the PHC (Fig. 4; $t=2.93, p=.007$ ). After adjusting for total brain volume and MDD status, an increase of one point on the RRS-RSQ scale was associated with an increase of $5.6 \mathrm{~mm}^{3}$ in PHC volume $\left(S E=1.92 \mathrm{~mm}^{3}\right)$. Autosegmented ERC volumes in the left hemisphere were found to be significantly smaller in depressed patients than in matched controls (ERC: $F=8.349, p=.0008$ ).

The total volume of the right hippocampus was significantly reduced in the MDD group $(F=10.090, p=.00421)$. Within the right hippocampus, the volumes of CA1 $[F=$

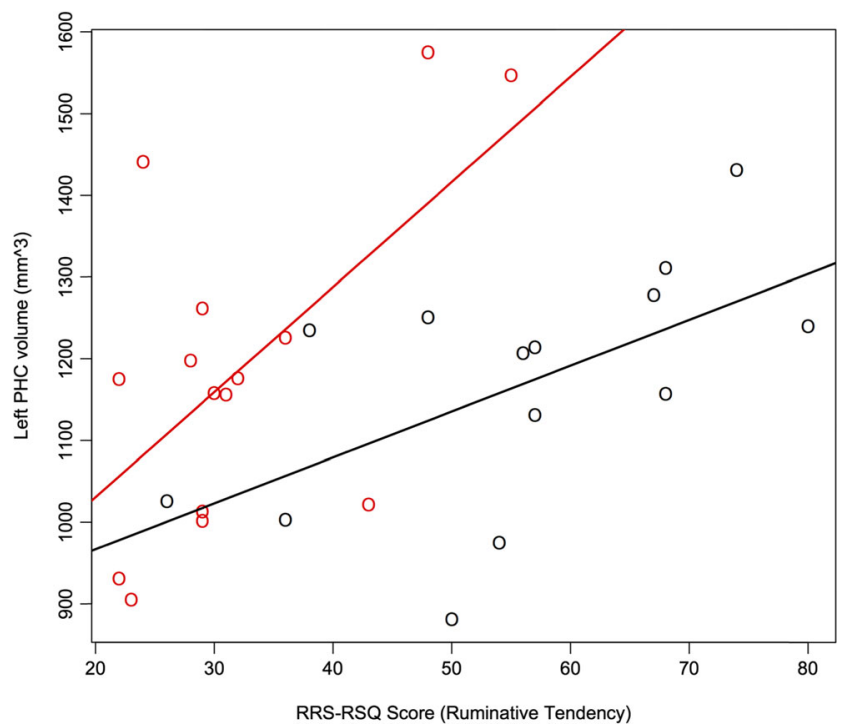

Fig. 4 Parahippocampal cortex (PHC) volume is significantly associated with ruminative tendency (RRS-RSQ score) after adjusting for total brain volume and MDD status $[\beta=5.6( \pm 1.92), p=.007]$. (gray [red in online version $]=$ healthy controls, black $=$ depressed patients)
15.153, $p=.00073$; effect size $=1.25(0.5,1.99)]$, CA2-3 $[F=14.297, p=.00097$; effect size $=1.2(0.47,1.94)]$, and CA4-DG $[15.637, p=.00063$; effect size $=1.25(0.51,1.99)]$ were significantly reduced in the MDD patients as compared to controls. The subiculum $(F=12.013, p=.0021)$ trended toward a significant volume reduction in MDD patients as compared to controls. Neither the total left hippocampus nor its subfields showed a volume reduction that was significant or trending at $p=.001$ (these results are summarized in the supplemental information).

\section{Discussion}

Previous studies indicated a direct relationship between mood and associative thinking, whereby negative mood constricts and positive mood broadens the breadth of associations. With this framework in mind, we hypothesized that contextual associative processing might be compromised in depression. To test this, we compared the brain activity and gray matter volumes of regions involved in the processing of contextual associations between depressed participants and a healthy control group. Consistent with our hypothesis, depressed patients displayed a pattern of activation and morphological characteristics that differed from the typical "context network" displayed in healthy participants.

Compared with healthy controls, depressed participants showed several activational differences in medial temporal lobe and prefrontal cortex regions: (1) A decreased differential activation in the PHC, (2) A greater differential activation in the ERC, and (3) A greater differential activation in the MFG (Fig. 2). We did not find statistically significant group differences in other areas of the context network such as the RSC and the MPFC. Alongside these results, our morphological analyses revealed several findings: (1) PHC volume was positively associated with ruminative tendency, (2) decreased left ERC volume in depressed participants, and (3) decreased volume in specific hippocampal subfields (CA1, CA2-3, CA4DG) in depressed participants.

According to previous accounts of contextual processing in healthy participants (Bar, 2007; Bar \& Aminoff, 2003; Bar et al., 2008), the PHC plays a crucial role in object recognition by using information about the current context frame (e.g., bowling alley) to generate expected associated stimuli (e.g., bowling ball, bowling pin) (Bar, 2004; Bar \& Ullman, 1996). Moreover, in regards to this framework, it has been shown that the RSC (Aminoff et al., 2008; Bar, 2007; Bar \& Aminoff, 2003) contains contextual representations that may be more abstract and prototypical (i.e., context frames), whereas the PHC seems more directly related to the specific appearance and physical properties of a context and its associated elements (and thus might contain more episodic versions of context frames). Thus, our finding of impaired PHC activity in 
depressed participants provides evidence for a possible impairment in the generation of contextual associates, which is in line with our overarching hypothesis.

It is also interesting that PHC volume was positively correlated with ruminative tendency as measured with the RRS questionnaire. Whether these volume differences can be interpreted as increased effort by this region to retrieve contextual associations is unclear. If so, then the possibility that this activation and morphological impairment may give rise to ruminative thought pattern is highly intriguing and is also in accord with our general hypothesis connecting negative mood and rumination to constricted associative thought.

The greater ERC activation in our MDD patients compared with controls was not predicted in advance. Nevertheless, when viewed alongside the decreased ERC volume in our MDD patients, this finding corroborates and enriches existing morphological data that point to ERC pathology in depression. For example, previous studies had shown decreases in left ERC cortical thickness in depressed participants (Tu et al., 2012), reductions in left ERC volume in females with treatment-resistant depression (Furtado, Maller, \& Fitzgerald, 2008), an inverse correlation between bilateral hippocampal-entorhinal volume and years since onset of depression in geriatric depressed patients (Bell-McGinty et al., 2002), and an association between late onset depression and smaller ERC volume in patients with atherosclerotic disease (Gerritsen et al., 2011).

A possible account of our findings may be that the increased ERC activation during contextual processing is related to the decreased activity in PHC. The ERC seems to act as a relay station between the prefrontal cortex and the hippocampus and has a role in novelty detection (Witter, Wouterlood, Naber, \& Van Haeften, 2000). It is believed to function as a buffer that holds multimodal sensory information and compares it with internal representations to detect "familiarity" versus "novelty" (Arnold, 1999; Lörincz \& Buzsáki, 2000), whereas the PHC had been proposed to make contextual associations available. The task instructions were to rate the commonality of the object in the environment, requiring a comparison of the object presented to similar representations in memory that are common to our environment. Perhaps in depression the lack of availability of such information fed to the hippocampus by the PHC induces this increased, compensatory activity in the ERC. These inter-relations between MTL structures should naturally be further investigated in order to validate these proposals. Our finding of increased ERC activity during contextual processing in depression and decreased activity in the PHC opens interesting new avenues both for research and for potential therapy approaches. To our knowledge, this is the first such demonstration of functional abnormalities in the ERC in depression.

Elucidating the role of the ERC in the pathophysiology of depression is increasingly important, because this region is among the first to show volume loss with preclinical Alzheimer's disease (AD) (Dickerson et al., 2001), and the ERC is a potential target area for intervention by brain stimulation techniques to improve memory and learning (Suthana et al., 2012). The link between AD and depression has been suggested by studies showing lifetime depression as a risk factor for AD (Dotson, Beydoun, \& Zonderman, 2010; Green et al., 2003), that reversible memory impairment during depressive episodes is specifically a risk factor for dementia (Sáez-Fonseca, Lee, \& Walker, 2007), and that exposure to abnormal levels of psychosocial stress is a common pathway leading to both neuropathologies (Aznar \& Knudsen, 2011). Given our findings and the literature reviewed here, interesting connections likely exist between the ERC and some characteristic hallmarks of MDD, such as anhedonia and negative recall bias. However, we did not specifically focus upon these traits when preparing our instruments and task design, so connecting these specific traits to our findings here may be overly speculative. These questions, although intriguing, will need to be addressed by future studies with a nuanced focus on these traits.

The finding that the MFG is differentially more engaged during contextual processing in depressed patients raises the question as to its functional significance during this task. The MFG, corresponding to BA 46d of DLPFC, has been associated with cognitive control in numerous studies (Koechlin, Ody, \& Kouneiher, 2003; Kouneiher, Charron, \& Koechlin, 2009) and abnormal activity in the left DLPFC has been previously documented in imaging studies of depression compared with healthy individual. For example, measures of activity observed during rest, as measured with PET or fMRI, point toward a reduction of baseline activity in DLPFC (Mayberg, Lewis, Regenold, \& Wagner, 1994; Rogers et al., 2004; Videbech et al., 2002). Additionally, behavioral studies of cognitive control tasks (e.g., working memory) typically associated with DLPFC activity show impairments in depression (Abas, Sahakian, \& Levy, 1990; Austin et al., 1999; Beats, Sahakian, \& Levy, 1996; Degl'Innocenti, Ågren, \& Bäckman, 1998; Elliott et al., 1996; Grant, Thase, \& Sweeney, 2001; Harvey et al., 2005; Sweeney, Kmiec, \& Kupfer, 2000), suggesting that depressed patients have abnormally functioning DLPFC activity during tasks demanding cognitive control. Although some neuroimaging studies of brain function in depressed patients during cognitive tasks have reported contradicting results (Harvey et al., 2005; Wagner et al., 2006; Walter, Wolf, Spitzer, \& Vasic, 2007), most have pointed to differential DLPFC hyperactivity in depressed participants.

In the present study, we observed similar higher activity of DLPFC specifically when participants were engaged in contextual processing, albeit overall reduced activity in DLPFC, as seen by the percent change from baseline (Table S1 in the supplemental information). Most models of brain 
abnormalities in depression emphasize the importance of the limbic-DLPFC reciprocal interactions in which limbic activity predominates DLPFC activity in depression (Mayberg et al., 1999). Whether this predominance is a result of a failed top-down inhibition by DLPFC or a reciprocal process originating in limbic areas and resulting in DLPFC hypo-activity is still not clear.

Our results displaying selective decreases in the volumes of the CA1-3 and DG subfields of the hippocampus are particularly intriguing. Reduction in hippocampal volume is one of the most replicated neurobiological changes found in MDD (McKinnon, Yucel, Nazarov, \& MacQueen, 2009), but comparatively few studies have investigated whether these volumetric reductions affect the hippocampus universally or if they are specific to certain subfields (Samuels, Leonardo, \& Hen, 2015). Hippocampal subfields are differentially activated for specific tasks and may be sensitive to specific environmental stimuli (Fanselow \& Dong, 2010; Thompson et al., 2008; Zhao et al., 2001), so elucidating volumetric reductions of individual subfields may provide insight into the pathogenic mechanisms of MDD (Small, Schobel, Buxton, Witter, \& Barnes, 2011). The CA1-3 and DG subfields are responsible for pattern completion and pattern separation, respectively (Guo et al., 2015), and they each express large numbers of receptors for stress-related hormones (e.g., glucocorticoids; Small et al., 2011). Since MDD is characterized by increased, chronic exposure to stress, the experience of MDD may lead to impairments in the structure, and likely the function, of these subfields. If pattern completion and separation are impaired, individuals may have limited ability to integrate new, positive experiences into their thought patterns or to form new, broader associations to break ruminative thought patterns. The first portion of this pathway has been supported by recent research displaying longitudinal decreases in the DG with increased depressive episodes (Treadway et al., 2015), but the second portion, connecting the DG, CA, and associative thinking, is more hypothetical still.

\section{Limitations}

The present study has several limitations. First, the sample size (15 participants in each group) is smaller than would be ideal. However, the MDD participants were all nonmedicated, removing the confounding effects of treatment on brain activation and morphology, which is a common limitation in studies of MDD. Second, the task used to assess contextual processing had no behavioral component apart from the commonality ratings of the objects and the reaction times for those ratings. Hence, the amount of engagement in the task could not be measured. However, given our previous experience with this task, there is little doubt about participants' actual engagement and adherence. Furthermore, this limitation should be regarded, keeping in mind the wide acceptance of the measurement of resting state activity and the conclusions drawn from such tasks about default network activity. In this sense, the task used in this study is much more constrained in measuring associative processing.

\section{Conclusions}

In summary, our fMRI results showed impaired brain activity during the processing of contextual information in acute nonmedicated depressed individuals compared with controls. Furthermore, we found that these individuals also have volumetric differences in regions connected with this contextual processing. Overall, these results suggest that the MTL structures of the PHC and the ERC are differentially affected by MDD, potentially causing ERC activity to increase in an attempt to increase the recollection of contextual memory.

Our study implicates a connection between mood and contextual processing, wherein the characteristic traits of MDD thought patterns - specifically, a ruminative tendency and narrow associative scope - are connected to measurable changes in both the activity and the structure of the context network. Combined with existing psychiatric knowledge of the symptomatology of MDD, our findings point at potentially important targets for both research on and the treatment of depressive disorders.

Author note This work was supported by National Institutes of Health Grant No. R01MH084940, and by the Israeli Center of Research Excellence in Cognition, Grant No. 51/11, both awarded to M.B. We thank the staff at the Depression Clinical and Research Program of the Massachusetts General Hospital for their skilled assistance in patient recruitment. E.V.H., R.L.T., and M.B. report no biomedical financial interests or potential conflicts of interest. For a list of lifetime disclosures for M.F., please see http://mghcme.org/faculty/faculty-detail/maurizio_fava.

\section{References}

Abas, M. A., Sahakian, B. J., \& Levy, R. (1990). Neuropsychological deficits and CT scan changes in elderly depressives. Psychological Medicine, 20, 507-552.

Aminoff, E., Schacter, D. L., \& Bar, M. (2008). The cortical underpinnings of context-based memory distortion. Journal of Cognitive Neuroscience, 20, 2226-2237. doi:10.1162/jocn.2008.20156

Andreasen, N. C. (1979). Thought, language, and communication disorders: I. Clinical assessment, definition of terms, and evaluation of their reliability. Archives of General Psychiatry, 36, 1315-1321.

Arnold, O. H. (1999). Schizophrenia-A disturbance of signal interaction between the entorhinal cortex and the dentate gyrus? The contribution of experimental dibenamine psychosis to the pathogenesis of schizophrenia: A hypothesis. Neuropsychobiology, 40, 21-32.

Austin, M. P., Mitchell, P., Wilhelm, K., Parker, G., Hickie, I., Brodaty, H., . . . Hadzi-Pavlovic, D. (1999). Melancholic depression: A distinct pattern of frontal impairment in melancholia. Psychological Medicine, 24, 73-85. 
Aznar, S., \& Knudsen, G. M. (2011). Depression and Alzheimer's disease: Is stress the initiating factor in a common neuropathological cascade? Journal of Alzheimer's Disease, 23, 177-193.

Bar, M. (2004). Visual objects in context. Nature Reviews Neuroscience, 5, 617-629. doi:10.1038/nrn1476

Bar, M. (2007). The proactive brain: Using analogies and associations to generate predictions. Trends in Cognitive Sciences, 11, 280-289. doi:10.1016/j.tics.2007.05.005

Bar, M. (2009a). A cognitive neuroscience hypothesis of mood and depression. Trends in Cognitive Sciences, 13, 456-463.

Bar, M. (2009b). The proactive brain: Memory for predictions. Philosophical Transactions of the Royal Society B, 364, 1235-1243.

Bar, M., \& Aminoff, E. (2003). Cortical analysis of visual context. Neuron, 38, 347-358. doi:10.1016/S0896-627300167-3

Bar, M., Aminoff, E., Mason, M., \& Fenske, M. (2007). The units of thought. Hippocampus, 17, 420-428.

Bar, M., Aminoff, E., \& Schacter, D. L. (2008). Scenes unseen: The parahippocampal cortex intrinsically subserves contextual associations, not scenes or places per se. Journal of Neuroscience, 28 , 8539-8544. doi:10.1523/JNEUROSCI.0987-08.2008

Bar, M., \& Ullman, S. (1996). Spatial context in recognition. Perception, 25, 343-352.

Baror, S., \& Bar, M. (2016). Associative activation and its relation to exploration and exploitation in the brain. Psychological Science, 27. 776-789. doi:10.1177/0956797616634487

Barsalou, L. W. (1999). Perceptual symbol systems. Behavioral and Brain Sciences, 22, 577-609. doi:10.1017/S0140525X99002149. disc. 609-66.

Beats, B. C., Sahakian, B. J., \& Levy, R. (1996). Cognitive performance in tests sensitive to frontal lobe dysfunction in the elderly depressed. Psychological Medicine, 26, 591-603.

Bell-McGinty, S., Butters, M. A., Meltzer, C. C., Greer, P. J., Reynolds, C. F., \& Becker, J. T. (2002). Brain morphometric abnormalities in geriatric depression: Long-term neurobiological effects of illness duration. American Journal of Psychiatry, 159, 1424-1427.

Cameron, H. A., \& Gould, E. (1994). Adult neurogenesis is regulated by adrenal steroids in the dentate gyrus. Neuroscience, 61, 203-209.

Davis, M. A. (2009). Understanding the relationship between mood and creativity: A meta-analysis. Organizational Behavior and Human Decision Processes, 108, 25-38.

Degl'Innocenti, A., Ågren, H., \& Bäckman, L. (1998). Executive deficits in major depression. Acta Psychiatrica Scandinavica, 97, 182-188.

Deichmann, R., Gottfried, J. A., Hutton, C., \& Turner, R. (2003). Optimized EPI for fMRI studies of the orbitofrontal cortex. NeuroImage, 19, 430-441.

Deichmann, R., Josephs, O., Hutton, C., Corfield, D. R., \& Turner, R. (2002). Compensation of susceptibility-induced BOLD sensitivity losses in echo-planar fMRI imaging. NeuroImage, 15, 120-135.

Dickerson, B. C., Goncharova, I., Sullivan, M. P., Forchetti, C., Wilson, R. S., Bennett, D. A., \& Beckett, L. A. (2001). MRI-derived entorhinal and hippocampal atrophy in incipient and very mild Alzheimer's disease. Neurobiology of Aging, 22, 747-754.

Dotson, V. M., Beydoun, M. A., \& Zonderman, A. B. (2010). Recurrent depressive symptoms and the incidence of dementia and mild cognitive impairment. Neurology, 75, 27-34.

Duncker, K. (1945). On problem-solving (L. S. Lees, Trans.). Psychological Monographs, 58(5, Whole No. 270), 1-113. doi:10.1037/h0093599

Elliott, R., Sahakian, B. J., McKay, A. P., Herrod, J. J., Robbins, T. W., \& Paykel, E. S. (1996). Neuropsychological impairments in unipolar depression: The influence of perceived failure on subsequent performance. Psychological Medicine, 26, 975-989.

Fanselow, M. S., \& Dong, H.-W. (2010). Are the dorsal and ventral hippocampus functionally distinct structures? Neuron, 65, 7-19. doi:10.1016/j.neuron.2009.11.031
Ferrer, R. A., Grenen, E. G., \& Taber, J. M. (2015). Effectiveness of Internet-based affect induction procedures: A systematic review and meta-analysis. Emotion, 15, 752-762. doi:10.1037 /emo0000035

Furtado, C. P., Maller, J. J., \& Fitzgerald, P. B. (2008). A magnetic resonance imaging study of the entorhinal cortex in treatment-resistant depression. Psychiatry Research: Neuroimaging, 163, 133-142.

Gerritsen, L., Comijs, H. C., van der Graaf, Y., Knoops, A. J. G., Penninx, B. W. J. H., \& Geerlings, M. I. (2011). Depression, hypothalamic pituitary adrenal axis, and hippocampal and entorhinal cortex volumes-The SMART Medea study. Biological Psychiatry, 70, 373 38. doi:10.1016/j.biopsych.2011.01.029

Grant, M. M., Thase, M. E., \& Sweeney, J. A. (2001). Cognitive disturbance in outpatient depressed younger adults: Evidence of modest impairment. Biological Psychiatry, 50, 35-43.

Green, R. C., Cupples, L. A., Kurz, A., Auerbach, S., Go, R., Sadovnick, D., . . . Griffith, P. A. (2003). Depression as a risk factor for Alzheimer disease: The MIRAGE Study. Archives of Neurology, 60, 753-759.

Guo, N., Gatchel, J., \& Sahay, A. (2015). The role of adult-born dentate granule neurons in the regulation of mood. In H. G. Kuhn \& A. J. Eisch (Eds.), Neural stem cells in development, adulthood and disease (pp. 41-52). New York, NY: Springer.

Harvey, P.-O., Fossati, P., Pochon, J.-B., Levy, R., LeBastard, G., Lehéricy, S., . . Dubois, B. (2005). Cognitive control and brain resources in major depression: An fMRI study using the $n$-back task. Neurolmage, 26, 860-869. doi:10.1016/j.neuroimage.2005.02.048

Isen, A. M., \& Daubman, K. A. (1984). The influence of affect on categorization. Journal of Personality and Social Psychology, 47, 1206 1217. doi:10.1037/0022-3514.47.6.1206

Isen, A. M., Daubman, K. A., \& Nowicki, G. P. (1987). Positive affect facilitates creative problem solving. Journal of Personality and Social Psychology, 52, 1122-1131. doi:10.1037/00223514.52.6.1122

Isen, A. M., Johnson, M. M., Mertz, E., \& Robinson, G. F. (1985). The influence of positive affect on the unusualness of word associations. Journal of Personality and Social Psychology, 48, 1413-1426.

Koechlin, E., Ody, C., \& Kouneiher, F. (2003). The architecture of cognitive control in the human prefrontal cortex. Science, 302, 11811185. doi:10.1126/science. 1088545

Kouneiher, F., Charron, S., \& Koechlin, E. (2009). Motivation and cognitive control in the human prefrontal cortex. Nature Neuroscience, $12,939-945$.

Lake, C. R. (2008). Disorders of thought are severe mood disorders: The selective attention defect in mania challenges the Kraepelinian dichotomy-A review. Schizophrenia Bulletin, 34, 109-117. doi:10.1093/schbul/sbm035

Lörincz, A., \& Buzsáki, G. (2000). Two-phase computational model training long-term memories in the entorhinal-hippocampal region. Annals of the New York Academy of Sciences, 911, 83-111.

Mandler, J. M., \& Johnson, N. S. (1976). Some of the thousand words a picture is worth. Journal of Experimental Psychology: Human Learning and Memory, 2, 529-554. doi:10.1037/0278-7393.2.5.529

Mason, M. F., \& Bar, M. (2012). The effect of mental progression on mood. Journal of Experimental Psychology: General, 141, $217-$ 221.

Mayberg, H. S., Liotti, M., Brannan, S. K., McGinnis, S., Mahurin, R. K., Jerabek, P. A., . . . Fox, P. T. (1999). Reciprocal limbic-cortical function and negative mood: Converging PET findings in depression and normal sadness. American Journal of Psychiatry, 156, $675-682$.

Mayes, A., Montaldi, D., \& Migo, E. (2007). Associative memory and the medial temporal lobes. Trends in Cognitive Sciences, 11, 126135.

McKinnon, M. C., Yucel, K., Nazarov, A., \& MacQueen, G. M. (2009). A meta-analysis examining clinical predictors of hippocampal volume 
in patients with major depressive disorder. Journal of Psychiatry and Neuroscience, 34, 41-54.

Mednick, M. T., Mednick, S. A., \& Jung, C. C. (1964). Continual association as a function of level of creativity and type of verbal stimulus. Journal of Abnormal and Social Psychology, 69, 511-515.

Mednick, M. T., Mednick, S. A., \& Mednick, E. V. (1964). Incubation of creative performance and specific associative priming. Journal of Abnormal and Social Psychology, 69, 84-88.

Minsky, M. (1975). A framework for representing knowledge. In P. H. Winston (Ed.), The psychology of computer vision (pp. 211-277). New York, NY: McGraw-Hill.

Moores, E., Laiti, L., \& Chelazzi, L. (2003). Associative knowledge controls deployment of visual selective attention. Nature Neuroscience, 6, 182-189. doi:10.1038/nn996

Nolen-Hoeksema, S. (2000). The role of rumination in depressive disorders and mixed anxiety/depressive symptoms. Journal of Abnormal Psychology, 109, 504-511.

Oliva, A., \& Torralba, A. (2001). Modeling the shape of the scene: A holistic representation of the spatial envelope. International Journal of Computer Vision, 42, 145-175.

R Development Core Team. (2011). R: A language and environment for statistical computing [Software]. Vienna, Austria: R Foundation for Statistical Computing. Retrieved from www.R-project.org

Rogers, M. A., Kasai, K., Koji, M., Fukuda, R., Iwanami, A., Nakagome, K., . . . Kato, N. (2004). Executive and prefrontal dysfunction in unipolar depression: A review of neuropsychological and imaging evidence. Neuroscience Research, 50, 1-11. doi:10.1016/j. neures.2004.05.003

Rosch, E., \& Mervis, C. B. (1975). Family resemblances: Studies in the internal structure of categories. Cognitive Psychology, 7, 573-605. doi:10.1016/0010-0285(75)90024-9

Sáez-Fonseca, J. A., Lee, L., \& Walker, Z. (2007). Long-term outcome of depressive pseudodementia in the elderly. Journal of Affective Disorders, 101, 123-129.

Samuels, B. A., Leonardo, E. D., \& Hen, R. (2015). Hippocampal subfields and major depressive disorder. Biological Psychiatry, 77, 210-211.

Sass, L., \& Pienkos, E. (2015). Beyond words: Linguistic experience in melancholia, mania, and schizophrenia. Phenomenology and the Cognitive Sciences, 14, 475-495. doi:10.1007/s11097-013-9340-0

Schank, R. C. (1975). Using knowledge to understand. In R. C. Schank \& B. L. Nash-Webber (Eds.), Theoretical issues in natural language processing (pp. 117-121). Stroudsburg, PA: Association for Computational Linguistics.

Small, S. A., Schobel, S. A., Buxton, R. B., Witter, M. P., \& Barnes, C. A. (2011). A pathophysiological framework of hippocampal dysfunction in ageing and disease. Nature Reviews Neuroscience, 12, 585601.

Suthana, N., Haneef, Z., Stern, J., Mukamel, R., Behnke, E., Knowlton, B., \& Fried, I. (2012). Memory enhancement and deep-brain stimulation of the entorhinal area. New England Journal of Medicine, 366, 502-551.

Sweeney, J. A., Kmiec, J. A., \& Kupfer, D. J. (2000). Neuropsychologic impairments in bipolar and unipolar mood disorders on the CANTAB neurocognitive battery. Biological Psychiatry, 48, 674684.

Thompson, C. L., Pathak, S. D., Jeromin, A., Ng, L. L., MacPherson, C. R., Mortrud, M. T., . . Puchalski, R. B. (2008). Genomic anatomy of the hippocampus. Neuron, 60, 1010-1021.

Travis, S., Coupland, N. J., Silversone, P. H., Huang, Y., Fujiwara, E., Carter, R., . . . Malykhin, N. V. (2015). Dentate gyrus volume and memory performance in major depressive disorder. Journal of Affective Disorders, 172, 159-164.

Treadway, M. T., Waskom, M. L., Dillon, D. G., Holmes, A. J., Park, M. T. M., Chakravarty, M. M., . . . Pizzagalli, D. A. (2015). Illness progression, recent stress, and morphometry of hippocampal subfields and medial prefrontal cortex in major depression. Biological Psychiatry, 77, 285-294. doi:10.1016/j.biopsych.2014.06.018

Treynor, W., Gonzalez, R., \& Nolen-Hoeksema, S. (2003). Rumination reconsidered: A psychometric analysis. Cognitive Therapy and Research, 27, 247-259.

Tu, P.-C., Chen, L.-F., Hsieh, J.-C., Bai, Y.-M., Li, C.-T., \& Su, T.-P. (2012). Regional cortical thinning in patients with major depressive disorder: A surface-based morphometry study. Psychiatry Research: Neuroimaging, 202, 206-213.

Videbech, P., Ravnkilde, B., Pedersen, T. H., Hartvig, H., Egander, A., Clemmensen, K., . . Rosenberg, R. (2002). The Danish PET/ depression project: Clinical symptoms and cerebral blood flow: A regions-of-interest analysis. Acta Psychiatrica Scandinavica, 106, $35-44$.

Wagner, G., Sinsel, E., Sobanski, T., Köhler, S., Marinou, V., Mentzel, H.J., . . Schlösser, R. G. M. (2006). Cortical inefficiency in patients with unipolar depression: An event-related fMRI study with the Stroop task. Biological Psychiatry, 59, 958-965.

Walter, H., Wolf, R. C., Spitzer, M., \& Vasic, N. (2007). Increased left prefrontal activation in patients with unipolar depression: An eventrelated, parametric, performance-controlled fMRI study. Journal of Affective Disorders, 101, 175-185.

Weiskopf, N., Hutton, C., Josephs, O., \& Deichmann, R. (2006). Optimal EPI parameters for reduction of susceptibility-induced BOLD sensitivity losses: A whole-brain analysis at $3 \mathrm{~T}$ and $1.5 \mathrm{~T}$. NeuroImage, 33, 493-504.

Witter, M. P., Wouterlood, F. G., Naber, P. A., \& Van Haeften, T. (2000). Anatomical organization of the parahippocampal-hippocampal network. Annals of the New York Academy of Sciences, 911, 1-24.

Zhao, X., Lein, E. S., He, A., Smith, S. C., Aston, C., \& Gage, F. H. (2001). Transcriptional profiling reveals strict boundaries between hippocampal subregions. Journal of Comparative Neurology, 441, 187-196. 